\title{
Disposición al estudio, autoeficacia y atribuciones causales en estudiantes universitarios chilenos
}

\section{Willingness to Study, Self-Efficacy and Causal Attributions in Chilean University Students}

Fabiola M. Sáez * iD Universidad de Concepción, Concepción, Chile. ORCID: https://orcid.org/0000-0002-7993-5356

Claudio E. Bustos (iD Universidad de Concepción, Concepción, Chile. ORCID: https://orcid.org/0000-0003-3478-9858

María V. Pérez Universidad de Concepción, Concepción, Chile. ORCID: https://orcid.org/0000-0002-3697-7978 Javier A. Mella Universidad Técnica Federico Santa María, Concepción, Chile.
ORCID: https://orcid.org/0000-0002-4288-142X

Karla A. Lobos (iD) Universidad Santo Tomás, Santiago, Chile. ORCID: https://orcid.org/0000-0002-0145-0041

Alejandro E. Díaz (iD) Universidad de Concepción, Concepción, Chile. ORCID: http://orcid.org/0000-0002-3090-5463

Recibido 01-09-17 Revisado 07-10-17 Aprobado 03-01-18 En línea 16-01-18

*Correspondencia

Email: famasade@gmail.com
Citar como:

Sáez F.M., Bustos, C.E., Pérez, M.V., Mella, J.A., Lobos, K.A., \& Díaz, A. E. (2018). Disposición al estudio, autoeficacia y atribuciones causales en estudiantes universitarios chilenos. Propósitos y Representaciones, 6(1), 199-245. doi: http:// dx.doi.org/10.20511/pyr2018.v6n1.179

(C) Universidad San Ignacio de Loyola, Vicerrectorado de Investigación, 2018

(cc) BY-NC-ND Este artículo se distribuye bajo licencia CC BY-NC-ND 4.0 Internacional (http://creativecommons.org/licenses/by-nc-nd/4.0/). 


\section{Resumen}

El objetivo de este estudio es: analizar la relación entre (1) Las estrategias disposición al estudio, (2) atribuciones causales (al esfuerzo, capacidad y causas externas) y (3) la percepción de autoeficacia que tienen los estudiantes sobre su capacidad de autorregular sus procesos de disposición al estudio. Método: Se aplicó un instrumento construido por los investigadores denominado Cuestionario de Autorregulación de la Disposición al Estudio a una muestra no probabilística por conveniencia de 695 estudiantes universitarios chilenos de 5 universidades de la provincia de Concepción. Resultados: Se encontraron correlaciones fuertes entre la autoeficacia para la autorregulación de la disposición al estudio y las estrategias de disposición al estudio ( $\mathrm{r}=0.54 \mathrm{a} \mathrm{r}=0.55)$. Las estrategias de disposición al estudio presentan correlaciones positivas y moderadas $(\mathrm{r}=0.38 \mathrm{a} \mathrm{r}=0.42)$ con las atribuciones causales de éxito al esfuerzo, débiles $(\mathrm{r}=0.15 \mathrm{a} \mathrm{r}=0.28)$ con las atribuciones causales de éxito a la habilidad como a las atribuciones causales de éxito a causas externas, y correlaciones negativas, de débiles a moderadas $(\mathrm{r}=-0.19 \mathrm{a}$ $\mathrm{r}=-0.38$ ), con las atribuciones causales de fracaso al esfuerzo, a la habilidad y a causas externas. Conclusiones: Los estudiantes con altos niveles de estrategias de disposición al estudio presentan creencias positivas acerca de la propia capacidad para autorregular sus procesos de disposición al estudio, realizan atribuciones causales de sus éxitos principalmente al esfuerzo y disminuyen las explicaciones de fracasos académicos a su esfuerzo, su capacidad y a causas externas.

Palabras clave: Aprendizaje autorregulado, disposición al estudio, autoeficacia, atribuciones causales, educación Superior. 


\section{Summary}

The aim of this study is to analyze the relationship between (1) willingness to study strategies, (2) causal attributions (to effort, ability and external causes) and (3) student's perception of self-efficacy about their ability to self-regulate their processes of willingness to study. Method: An instrument built by the researchers called Willingness to the Study Self-Regulation Questionnaire was applied to a non-probabilistic convenience sample of 695 Chilean university students from 5 universities of the Province of Concepción. Outcomes: Strong correlations were found between selfefficacy for the willingness to study self-regulation and the willingness to study strategies ( $r=0.54$ to $r=0.55$ ). the willingness to study strategies had positive and moderate correlations ( $\mathrm{r}=0.38$ to $\mathrm{r}=0.42$ ) with causal attributions for success to effort, weak correlations ( $r=0.15$ to $r=0.28)$ with causal attributions for success to ability and to external factors, and negative weak to moderate correlations ( $r=-0.19$ to $r=-0.38$ ) with causal attributions for failure to effort, ability, and external factors. Conclusions: Students with high levels of willingness to study strategies show positive beliefs about their own ability to self-regulate their processes of willingness to study, they make causal attributions for their success mainly to effort, and they attribute their academic failure less and less to ability and external factors.

Keywords: Self-regulated learning, disposition to study, self-efficacy, causal attributions, Higher Education. 


\section{Introducción}

Los procesos de autorregulación del aprendizaje en estudiantes universitarios son fundamentales para el auto monitoreo de las metas, motivaciones y emociones en la realización de las tareas académicas (Garello \& Rinaudo, 2012)the student constructs significant learning and usually obtains good academic performance. Self-regulated learning implies a social aspect that includes interactions with peers and teachers who act as co-regulators of learning. The teachers would be the persons in charge of constructing social support inside the classroom, for example creating opportunities for peer collaboration in the accomplishment of the tasks. The notion of distributed cognition allows us to express that we do not think or learn alone, but by collaborating with other people and using cultural tools. We proposed to study self-regulated learning processes and distributed cognition in university students by means of the implementation of two studies of design where 172 students from the Universidad Nacional de Rlu00edo Cuarto participated in 2007 and 2008. Across the activities and implemented protocols we could analyze the aspects of tasks that favor the students' processes of self-regulation of their performances and their participation in collaborative processes with their partners. Such aspects would be: demands for reflection on problems that they can find in their professional role, tasks that imply some level of control and evaluation, collaborative work, instances of integration of previous knowledge, out-of-school tasks, instances of feedback on the tasks, tasks of metacognitive and motivational reports and tasks with possibilities of review. (English. Desempeñan un papel importante en el éxito académico (Tauber, Dunlosky, Rawson, Wahlheim, \& Jacoby, 2012; Zimmerman, Kitsantas, \& Campillo, 2005), favoreciendo la consecución de los logros académicos y previniendo el fracaso académico (Schober et al., 2015). La incapacidad de gestionar y autodirigir el propio proceso de aprendizaje se ha identificado como una de las principales causas del fracaso en los estudiantes de todos los niveles (Vives-Varela, Durán-Cárdenas, Varela-Ruíz, \& Fortoul, 2014). Este déficit de procesos para realizar autorregulación del aprendizaje es un 
factor principal del fracaso académico particularmente en los estudiantes de Educación Superior (Carranza \& Apaza, 2015; Tuckman, 2003; Tuckman \& Kennedy, 2011).

La autorregulación del aprendizaje implica una disposición activa del estudiante a partir de la puesta en marcha de una serie de habilidades que involucran observar, vigilar y controlar los propios pensamientos, comportamientos y motivaciones para lograr un aprendizaje efectivo (VivesVarela et al., 2014). Para ser un aprendiz competente es necesario ser un participante autorregulado, es decir, intencionado y activo, capaz de iniciar y dirigir el propio aprendizaje y no un aprendiz reactivo (Vives-Varela et al., 2014).

Existen diferentes modelos de autorregulación con respaldo teórico y empírico (Monique Boekaerts \& Corno, 2005; Hadwin \& Oshige, 2011; Pintrich, 2000; Winne \& Hadwin, 1998; Zimmerman, 2000), todos los autores coinciden en que la autorregulación del aprendizaje es un proceso cíclico. En términos generales, se consideran tres fases: disposición, desempeño y evaluación (Panadero, 2017). La primera fase, de disposición o preparación, implica un proceso de activación, análisis de tareas, planificación, establecimiento de objetivos y metas. La fase de desempeño considera la ejecución de la tarea. En la tercera fase, de evaluación, el estudiante regula y adapta para futuras actuaciones (Puustinen \& Pulkkinen, 2001).

La importancia de la fase de disposición o preparación, radica en que en esta fase se despliega y pone en marcha las siguientes fases del proceso. Estudiar requiere que el alumno posea habilidades académicas, pero además es necesaria su disposición ante la actividad de estudio (Barrera, Donolo, $\&$ Rinaudo, 2008). Es necesario que los aprendices tomen conciencia de la forma de abordar una actividad de aprendizaje, y de qué modo beneficiarse más de ellas, por ejemplo, gestionando mejor su tiempo o incorporando nuevas estrategias para alcanzar el éxito académico (Durán-Aponte \& Pujol, 2013). La disposición al estudio integra diversas estrategias y creencias de generación de actitud, estado emocional, motivación y planificación para que 
una tarea se lleve a cabo y con éxito (Pérez, Valenzuela, Díaz, GonzálezPienda, \& Núñez, 2011, 2013; Yan, Khanh-Phuong, \& Bjork, 2014).

Las estrategias y creencias de disposición al estudio contribuyen a generar una motivación, actitud y estado emocional adecuados para la resolución de tareas de aprendizaje (Pérez et al., 2011). Las estrategias de disposición al estudio incluyen: (1) establecimiento de objetivos académicos, (2) gestión del tiempo académico y (3) organización de los recursos materiales y ambientales. Las creencias de disposición al estudio son: (1) autoeficacia para la autorregulación de la disposición al estudio y (2) atribuciones causales en la autorregulación de la disposición al estudio (Elvira-Valdéz \& Pujol, 2014; M. Pérez et al., 2011; Rosário et al., 2007; Zimmerman, 2008).

Dentro de las estrategias de disposición al estudio, el establecimiento de objetivos es precisar un propósito de comportamiento para alcanzar resultados de aprendizaje dentro de un período de tiempo determinado (Bloom, 2013; Cleary, Callan, \& Zimmerman, 2012); la gestión del tiempo académico es el conocimiento y utilización de los espacios temporales requeridos para efectuar una tarea, y al orden de ellos para el logro de los objetivos de aprendizaje (Oettingen, Kappes, Guttenberg, \& Gollwitzer, 2015)we asked students to apply MCII to a pressing academic problem and assessed how they scheduled their time for the upcoming week. MCII participants scheduled more time than control participants who in their thoughts either reflected on similar contents using different cognitive proce- dures (contentcontrol group; y la organización de recursos materiales y ambientales es el ordenamiento de recursos materiales necesarios para atender a la tarea de aprendizaje y el ordenamiento de recursos ambientales para la adaptación de las condiciones del ambiente con las que se dispone para realizar la tarea de aprendizaje (García-Ros \& Pérez-González, 2011).

Respecto de las creencias, las atribuciones causales en la autorregulación del estudio se entiende como el conjunto de creencias y causas que se perciben como responsables de la autorregulación del estudio, permiten dar cuenta de las representaciones que hacen los sujetos respecto del esfuerzo emprendido 
y del resultado alcanzado en una tarea, estableciendo una relación causal entre ambos (A. Fernández, Arnaiz, Mejía, \& Barca, 2015); y la autoeficacia para la disposición al estudio se define como las creencias sobre la propia capacidad en la utilización de estrategias de estudio en un dominio específico (Bandura, 2012; Wang, Shannon, \& Ross, 2013).

El presente estudio busca comprender cómo están relacionadas las estrategias y las creencias de la fase de disposición al estudio. Si bien se han estudiado relaciones de variables de disposición con otros variables de aprendizaje, como por ejemplo entre estrategias de disposición al aprendizaje y enfoques de aprendizaje (Pérez et al., 2011), o la relación entre gestión del tiempo, regulación del esfuerzo, incentivos y rendimiento académico (Broc, 2011), aún no se ha encontrado un estudio que relacione cada una de las estrategias y creencias de la fase de disposición al estudio entre ellas en estudiantes universitarios. Al parecer se centran en la relación entre estrategias o creencias del proceso de autorregulación, pero no en ambas, y los estudios que han analizado las relaciones entre estrategias y creencias de autorregulación, no se han centrado sólo en la fase de disposición, o de forma específica en los procesos de una de las fases de autorregulación, sino consideran diferentes estrategias y creencias de autorregulación en general. La literatura recomienda analizar relaciones que aporten a los procesos específicos del aprendizaje autorregulado (Pool-Cibrián \& MartínezGuerrero, 2013).

El objetivo del estudio es analizar la relación que existe entre las estrategias de disposición al estudio (establecimiento de objetivos académicos, gestión del tiempo de estudio y organización de recursos materiales y ambientales), las atribuciones causales (al esfuerzo, capacidad y causas externas) y la percepción de autoeficacia que tienen los estudiantes sobre su capacidad de autorregular sus procesos disposición al estudio. La importancia de conocer la relación entre estas variables, se justifica en aportar a la comprensión de los procesos específicos del aprendizaje autorregulado (Pool-Cibrián \& Martínez-Guerrero, 2013), demostrado ser una variable clave en el éxito 
académico de los estudiantes especialmente en primeros años de experiencia universitaria. Esto puede servir para implementar intervenciones en estudiantes de Educación Superior que tengan por propósito la mejora de competencias para la autorregulación del aprendizaje.

\section{Método}

\section{Diseño}

Para estudiar la relación entre las estrategias de disposición al estudio, las creencias de autoeficacia para la autorregulación de la disposición al estudio y las atribuciones causales en la autorregulación del estudio, se utilizó un diseño descriptivo-correlacional, de corte transversal.

\section{Participantes}

Se encuestó a un total de 694 estudiantes seleccionados a través de un muestreo de tipo no probabilístico por accesibilidad, provenientes de 14 programas de 5 universidades de la Provincia de Concepción en Chile.

Los cuestionarios fueron aplicados en asignaturas del primer semestre de distintos niveles: $22.05 \%$ de primer año, $37.03 \%$ de segundo, $15.13 \%$ de tercero, $19.60 \%$ de cuarto y $6.20 \%$ de quinto año. La media de edad de los sujetos fue de 21.18 (DE=2.78), con un mínimo de 17 y un máximo de 36. Con respecto al sexo, 353 fueron hombres (50.79\%) y 340 fueron mujeres (48. 92\%).

\section{Instrumento}

Se aplicó un cuestionario de autoinforme compuesto de 3 escalas, correspondientes a (a) estrategias de disposición al estudio, (b) atribuciones causales y (c) autoeficacia para la disposición al estudio. Cada una de estas 
escalas se construyó a partir de otros instrumentos disponibles previamente (García-Ros \& Pérez-González, 2011; Inglés, Rodríguez-Marín, \& GonzálezPienda, 2008; Pérez \& Delgado, 2006) fueron validados en el contexto de esta investigación siguiendo las directrices de diseño, construcción y adaptación de escalas de estrategias de estudio y autorregulación (Castañeda, Pineda, Gutierrez, Romero, \& Peñaloza, 2010; Muñiz, Elosua, \& Hambleton, 2013). Todo el cuestionario cuenta con evidencias de validez de contenido y de proceso, obtenido de la revisión por jueces expertos, y entrevista cognitiva, respectivamente.

La Escala de Estrategias de Disposición al Estudio, fue construida a partir del instrumento Time Management Behavior Questionnaire (García-Ros \& Pérez-González, 2011; Macan, Shahani, Dipboye, \& Peek, 1990). La escala, compuesta de 31 ítems, mide el uso de estrategias a través de tres subescalas: establecimiento de objetivos académicos (10 ítems, $\alpha$ de Cronbach $=0.82$ ), gestión del tiempo académico ( 11 ítems, $\alpha=0.82$ ), y organización de recursos materiales y ambientales ( 10 ítems, $\alpha=0.70$ ). Se pregunta usando la consigna "con qué frecuencia utilizo las siguientes estrategia" y se responde en escala Likert donde 1 es "nunca" y 5 es "siempre".

La Escala de Atribuciones Causales en la Autorregulación de la Disposición al Estudio corresponde a una adaptación de la Sydney Attribution Scale (Inglés et al., 2008; Marsh, 1984)508 college students. Factor analysis identified six factors: Success/Ability, Success/Effort, Success/External Causes, Failure/Ability, Failure/Effort, and Failure/External Causes. Success and failure factors accounted for an adequate percentage of the variance. Internal consistency was acceptable, similar in the success scales and in the failure scales, and higher in the internal scales than in the external scales. The results also showed a clear predictable pattern of relationships between dimensions of self-attribution, and between these dimensions and several measures of general self-efficacy, intrinsic motivation, extrinsic motivation, satisfaction with the studies, satisfaction with performance, and satisfaction with knowledge, which supports the construct validity of the SAS. 
(English. Evalúa la tendencia o estilo de los estudiantes a la hora de realizar autoatribuciones de éxito o fracaso a determinadas causas en el proceso de autorregulación del estudio. En total, posee 30 ítems, y se subdivide en 6 subescalas, cada una con 5 ítems: atribuciones de éxito al esfuerzo $(\alpha=0.84)$, la habilidad $(\alpha=0.81)$ o factores externos $(\alpha=0.35)$; y atribuciones causales de fracaso al esfuerzo $(\alpha=0.82)$, la habilidad $(\alpha=0.78)$ o factores externos $(\alpha=0.59)$. Se pregunta usando la consigna "en qué medida me sucede a mí" y se responde en escala Likert donde 1 es falso y 5 es verdadero.

La Escala de Autoeficacia para la Autorregulación del Estudio corresponde a una adaptación del Inventario de Autoeficacia para el Estudio (Pérez \& Delgado, 2006). Mide las creencias del estudiante sobre su propia capacidad para autorregular su proceso de estudio. Es un unidimensional y tiene 9 ítems. Se pregunta usando la consigna "creo que puedo utilizar la siguiente estrategia" y se responde en escala Likert donde 1 es nada seguro y 5 es muy seguro. Presenta un alfa de Cronbach de $\alpha=0.84$.

\section{Procedimiento.}

La aplicación del instrumento se realizó el primer semestre de 2016. Se contactó a diferentes autoridades de las 5 universidades, quienes indicaron que cursos podían ser partícipes de la investigación. Se contactó a los docentes de estos cursos, para coordinar la forma de aplicación de los instrumentos; los docentes informaron de la investigación con antelación a sus alumnos. En el día planificado para cada aplicación, se entregaron los consentimientos informados a los alumnos y se entregó el cuestionario a quienes aceptaban participar. El tiempo de aplicación del cuestionario no superó los 30 minutos por persona.

Una vez recolectada la información, se completó la base de datos en Microsoft Excel y se realizaron los análisis de datos. Esta investigación fue aprobada por el Comité Ética y Bioética del Departamento de Psicología de la Universidad de Concepción, Chile. 


\section{Análisis de Datos}

Se estudió de forma descriptiva la muestra, obteniendo los estadísticos medias y desviaciones estándar para cada variable. Posteriormente, se graficó la relación entre cada par de variables para verificar la linealidad de la relación. Comprobada ésta, se calculó la correlación de Pearson entre las variables de estudio; se analizó en primer lugar la relación existente entre las dimensiones de las distintas escalas, para después analizar la relación existente entre las dimensiones de distintas escalas. Todos los análisis se realizaron utilizando el software estadístico R, versión 3.3.1.

\section{Resultados}

En la tabla 1 se presentan los estadísticos de las escalas del Cuestionario de Disposición al Estudio. Al analizar las medias, se puede observar que el uso de las estrategias de disposición se encuentra en el rango entre "algunas veces" (Gestión del Tiempo Académico, M=2.94) y “muchas veces" (Organización de Recursos Materiales y Ambientales, $M=3.67$ ). En general, existe un mayor acuerdo con las afirmaciones sobre atribuciones causales al éxito que aquellas relacionadas al fracaso; dentro de ambas, el promedio es mayor en las atribuciones al esfuerzo ( $\mathrm{M}=3.2$ para éxito, $\mathrm{M}=2.72$ para fracaso). Las afirmaciones que los participantes consideran menos relacionados a sus creencias son las atribuciones causales de fracaso a la habilidad $(\mathrm{M}=1.79)$ y factores externos $(\mathrm{M}=1.97)$. La escala de autoeficacia para la autorregulación del estudio presenta una media alta, de 3.92, cercano a la respuesta "bastante seguro". La asimetría y curtosis de todas las variables, excepto la atribución causal de fracaso a la habilidad, se encuentran dentro del rango -1 a +1 , lo que indica variables con distribución cercanas a la normal. 


\section{Tabla 1.}

Descriptivos de las escalas del Cuestionario de Disposición al Estudio.

\begin{tabular}{lcccccc}
\hline Escalas & M & DE & Min & Max & asimetría & curtosis \\
\hline Estrategias de disposición al estudio & & & & & & \\
EOA & 3.34 & 0.58 & 1.50 & 5.00 & -0.20 & 0.16 \\
GTA & 2.94 & 0.67 & 1.18 & 4.91 & 0.14 & -0.32 \\
ORMA & 3.67 & 0.57 & 1.50 & 5.00 & -0.43 & .00 \\
Atribuciones causales de éxito & & & & & & \\
Esfuerzo & 3.22 & 0.82 & 1.00 & 5.00 & -0.18 & -0.32 \\
Habilidad & 2.98 & 0.73 & 1.00 & 5.00 & 0.10 & -0.19 \\
Factores externos & 2.65 & 0.54 & 1.00 & 4.60 & 0.20 & 0.18 \\
Atribuciones causales de fracaso & & & & & & \\
Esfuerzo & 2.72 & 0.94 & 1.00 & 5.00 & 0.24 & -0.79 \\
Habilidad & 1.79 & 0.73 & 1.00 & 4.60 & 1.02 & 0.59 \\
$\quad$ Factores externos & 1.97 & 0.64 & 1.00 & 4.40 & 0.50 & -0.16 \\
Autoeficacia & 3.92 & 0.65 & 1.56 & 5.00 & -0.57 & 0.35 \\
\hline
\end{tabular}

Nota: $n=694$. EOA: Establecimiento de objetivos académicos. GTA: Gestión del tiempo académico. ORMA: Organización de recursos materiales y ambientales. Todas las escalas están en el rango entre 1 y 5.-

En la tabla 2 se observan las correlaciones entre las escalas del Cuestionario de Disposición al Estudio. Todas las correlaciones son estadísticamente significativas, excepto la existente entre Atribuciones al éxito por factores externos y Atribución al fracaso por habilidad $(\mathrm{r}=-.01)$.

Los distintos tipos de estrategias de disposición al estudio, así como los tipos de atribuciones al éxito y fracaso están positivamente relacionadas entre sí. Específicamente, la relación más fuerte se observa entre las estrategias de gestión del tiempo académico y establecimiento de objetivos académicos $(\mathrm{r}=0.67)$; seguida por la relación entre la organización de recursos materiales y ambientales con establecimiento de objetivos académicos $(\mathrm{r}=0.55)$, y con gestión del tiempo académico $(\mathrm{r}=0.61)$. Las atribuciones causales al fracaso muestran relaciones de moderadas a fuertes (entre $\mathrm{r}=.45 \mathrm{y} \mathrm{r}=.66$ ), en tanto 
que las atribuciones causales de éxito están moderadamente relacionadas entre sí (entre $\mathrm{r}=0.41$ y r=.32).

Entre las atribuciones de éxito y fracaso se observan principalmente relaciones negativas, desde insignificantes a fuertes. Si bien las correlaciones más fuertes se observan entre atribuciones de éxito y fracaso del mismo tipo - atribuciones causales de éxito y fracaso al esfuerzo ( $\mathrm{r}=-.64)$ y entre las atribuciones causales de éxito y fracaso a la habilidad ( $\mathrm{r}=-.49)$ - la relación entre atribuciones de éxito y fracaso por causas externas es insignificante $(\mathrm{r}=.08)$. Las atribuciones causales de éxito a la habilidad presentan relaciones moderadas con el resto de las atribuciones de fracaso al esfuerzo, en tanto que las atribuciones causales de éxito al esfuerzo y a factores externos se asocian de forma débil o insignificante.

Las estrategias de disposición al estudio presentan correlaciones positivas y fuertes con las atribuciones causales de éxito al esfuerzo (entre $\mathrm{r}=0.48 \mathrm{y}$ $\mathrm{r}=0.42$ ) y correlaciones positivas, pero débiles, con las atribuciones causales de éxito a la habilidad y factores externos (entre $\mathrm{r}=0.15 \mathrm{y} \mathrm{r}=0.28$ ). La relación de las estrategias de disposición con las atribuciones causales de fracaso son negativas, entre débiles y moderadas (entre $\mathrm{r}=-0.19$ y r=-0.38).

La autoeficacia presenta correlaciones fuertes y positivas con las distintas estrategias de disposición al estudio (entre $\mathrm{r}=0.54 \mathrm{y} \mathrm{r}=0.55$ ); correlaciones positivas de débil a moderadas con las atribuciones causales de éxito al esfuerzo, la habilidad y factores externos (entre $r=0.13$ y $\quad r=0.32$ ). Finalmente, la autoeficacia presenta correlaciones débiles y negativas con las atribuciones causales de fracaso al esfuerzo, la habilidad y factores externos (entre $\mathrm{r}=-0.24$ y $\mathrm{r}=-0.25$ ). 
Tabla 2.

Correlaciones entre escalas del Cuestionario de Disposición al Estudio.

\begin{tabular}{llllllllll}
\hline Escala & GTA & ORMA & AEE & AEH & AEFE & AFE & AFH & AFFE & AE \\
\hline EOA & $0.67^{* *}$ & $0.55^{* *}$ & $0.38^{* *}$ & $0.28^{* *}$ & $0.21^{* *}$ & $-0.31^{* *}$ & $-0.19^{* *}$ & $-0.22^{* *}$ & $0,54^{* *}$ \\
GTA & $1^{* *}$ & $0.61^{* *}$ & $0.42^{* *}$ & $0.23^{* *}$ & $0.18^{* *}$ & $-0.38^{* *}$ & $-0.19^{* *}$ & $-0.23^{* *}$ & $0,55^{* *}$ \\
ORMA & & $1^{* *}$ & $0.42^{* *}$ & $0.21^{* *}$ & $0.15^{* *}$ & $-0.33^{* *}$ & $-0.25^{* *}$ & $-0.23^{* *}$ & $0,55^{* *}$ \\
AEE & & & $1^{* *}$ & $0.41^{* *}$ & $0.41^{* *}$ & $-0.62^{* *}$ & $-0.21^{* *}$ & $-0.11^{* *}$ & $0,32^{* *}$ \\
AEH & & & & $1^{* *}$ & $0.32^{* *}$ & $-0.43^{* *}$ & $-0.49^{* *}$ & $-0.34^{* *}$ & $0,22^{* *}$ \\
AEFE & & & & & $1^{* *}$ & $-0.16^{* *}$ & $-0.01^{* *}$ & $0.08^{*}$ & $0,13^{* *}$ \\
AFE & & & & & & $1 * *$ & $0.48^{* *}$ & $0.45^{* *}$ & $-0,24^{* *}$ \\
AFH & & & & & & & $1 * *$ & $0.66^{* *}$ & $-0,23^{* *}$ \\
AFFE & & & & & & & & $1 * *$ & $-0,25^{* *}$ \\
\hline
\end{tabular}

Nota: N=694. EOA: Establecimiento de objetivos académicos. GTA: Gestión del tiempo académico. ORMA: Organización de recursos materiales y ambientales. AEE: Atribuciones causales de éxito al esfuerzo. AEH: Atribuciones causales de éxito a la habilidad. AEFE: Atribuciones causales de éxito a factores externos. AFE: Atribuciones causales de fracaso al esfuerzo. AFH: Atribuciones causales de fracaso a la habilidad. AFFE: Atribuciones causales de fracaso a factores externos. AE: Autoeficacia en la autorregulación de la disposición al estudio.

$* * p<.01 * p<.05$

\section{Discusión}

El presente estudio se planteó como objetivo analizar la relación que existe entre las estrategias de disposición al estudio (establecimiento de objetivos académicos, gestión del tiempo de estudio y organización de recursos materiales y ambientales), las atribuciones causales (al esfuerzo, capacidad y causas externas) y la percepción de autoeficacia que tienen los estudiantes sobre su capacidad de autorregular sus procesos disposición al estudio.

El estudio de la relación entre las estrategias de disposición al estudio mostró relación significativa, fuerte y positiva entre el establecimiento de objetivos académicos, la gestión del tiempo académico y la organización de recursos materiales y ambientales. Este resultado, al igual que investigaciones previas, refuerza que los estudiantes que plantean objetivos de estudio, 
también gestionan el tiempo dedicado a estudiar y preparan sus materiales y el lugar para el estudio (Fernández et al., 2013).

La atribución de éxito al esfuerzo, se asocia de manera negativa y fuerte con la atribución de fracaso al esfuerzo, no existiendo relación de importancia con las atribuciones de fracaso a la habilidad y a factores externos; es decir, el estudiante que está de acuerdo con que su éxito se debe al esfuerzo también afirmará que su fracaso no se debe a la falta de esfuerzo, pudiendo atribuirse el fracaso tanto a la habilidad como a factores externos. En cambio, si bien la atribución de éxito a la habilidad se asocia principalmente a menor atribución de fracaso a la habilidad, también se relaciona con una menor atribución de fracaso al esfuerzo y factores externos; esto implica que cualquier afirmación relacionada al fracaso tiende a considerarse falsa, lo que podría explicar al considerar que el fracaso no es alternativa. A su vez, la atribución de éxito a factores externos presenta relaciones muy débiles con las atribuciones al fracaso; esto es, un alumno que esté de acuerdo con qué su éxito se debe a factores fuera de su control, no es posible predecir a qué atribuirá su fracaso. Estos resultados son similares a los encontrados en investigaciones previas realizadas con estudiantes universitarios de primer año (Fernández et al., 2015).

Respecto de las estrategias de disposición al estudio y las atribuciones causales de éxito al esfuerzo se encontraron correlaciones medias y positivas; mientras que con las atribuciones causales de éxito a la habilidad y factores externos son débiles. Es decir, los estudiantes que se disponen a estudiar y establecen objetivos académicos, gestionan su tiempo y organizan los materiales y lugar de estudio, hacen atribuciones de su éxito en autorregular su estudio a su esfuerzo y en menor medida a la habilidad y factores externos. (Heikkila, Niemivirta, Nieminen, \& Lonka, 2011). Un estudio presentó resultados similares, demostrando que el uso de estrategias de disposición se asocia con la atribución adecuada de las causas de los resultados académicos, donde los estudiantes atribuyen sus fracasos a causas internas y controlables (Pérez et al., 2013). 
La autoeficacia para la autorregulación de la disposición al estudio y las estrategias de disposición al estudio se asocian de manera fuerte y positiva, resultado que es consistente con otras investigaciones que han mostrado que existe relación entre la autoeficacia percibida y estrategias de autorregulación del aprendizaje (Alegre, 2014; Pool-Cibrián \& Martínez-Guerrero, 2013; Sevari \& Kandy, 2011; Yusuf, 2011; Zimmerman, Bandura, Martinez-pons, Zimmerman, \& Martinez-pons, 1992). La relación de la autoeficacia para la autorregulación de la disposición es positiva y moderada con las atribuciones causales de éxito, y negativa y débil con las atribuciones causales de fracaso, lo cual es consistente con los resultados disponibles en la literatura en estudiantes de secundaria y estudiantes universitarios (Becerra-González \& Reidl, 2015; García-Fernández et al., 2016).

Una fortaleza de este estudio es que explora la relación entre las atribuciones causales de fracaso y las atribuciones de éxito, el uso de estrategias y la autoeficacia. Si bien en la literatura se ha estudiado la relación entre las atribuciones al éxito y al fracaso con la autoeficacia y el rendimiento, en general sólo se estudian las atribuciones relacionadas al éxito (Erten \& Burden, 2014).

Como limitación del estudio, el diseño no permite realizar otro tipo de análisis entre las variables de estudio. Al tratarse de una investigación de tipo correlacional transversal, no es posible establecer conclusiones de causalidad entre las variables estudiadas. En el futuro, sería interesante considerar realizar estudios longitudinales prospectivos, que permitan el uso de modelos de ecuaciones estructurales para analizar causalidad. También otra limitación del estudio es su empleo exclusivo de medidas de autoinforme, por lo tanto estos resultados se basan en la declaración del uso de estrategias y creencias que el estudiante informa.

Estos hallazgos son relevantes para la futura investigación en el desarrollo de programas en la interacción en el aula que buscan ayudar a los estudiantes a ser autorregulados y lograr el éxito académico, fomentando el uso de estrategias para establecer objetivos académicos (Chi-Tung \& 
Ruey-Gwo, 2011; Hadwin \& Webster, 2013), gestionar el tiempo académico (Gaeta \& Cavazos, 2016), organizar sus recursos materiales y ambientales en instituciones de Educación Superior (Alvarado, Vega, Cepeda, \& Del Bosque, 2014). Las estrategias de disposición, a diferencia de las atribuciones de éxito y fracaso y la autoeficacia para la regulación del estudio, son directamente entrenables a través de diferentes estrategias didácticas o metodológicas (Torrano, Fuentes, \& Soria, 2017). Por lo tanto, es justificable el foco en la construcción y aplicación de programas de intervención que busquen mejorar las estrategias de disposición, ya que de acuerdo a los resultados de este estudio y a la literatura previa, se asocian positivamente a la autoeficacia para autorregular procesos de disposición al estudio y a las atribuciones causales del éxito al esfuerzo y, en menor medida, a la habilidad y/o factores externos. Estas estrategias de disposición al estudio deben ser fomentadas por los profesores en el contexto de aula, para facilitar el desarrollo de competencias de autorregulación del aprendizaje (Chilca, 2017).

\section{Referencias}

Alegre, A. (2014). Autoeficacia académica, autorregulación del aprendizaje y rendimiento académico en estudiantes universitarios iniciales. Propósitos y Representaciones, 2(1), 79-100. Doi: https://doi. org/10.20511/pyr2014.v2n1.54

Alvarado, I., Vega, Z., Cepeda, M., \& Del Bosque, A. (2014). Comparación de estrategias de estudio y autorregulación en universitarios. Revista Electronica de Investigacion Educativa, 16(1), 137-148.

Bandura, A. (2012). On the functional properties of perceived self-efficacy revisited. Journal of Management, 38(1), 9-44. Doi: https://doi. org/10.1177/0149206311410606

Barrera, M., Donolo, D., \& Rinaudo, M. (2008). Ritmo de estudio y trayectoria universitaria. Anales de Psicologia, 24(1), 9-15.

Becerra-González, C., \& Reidl, L. (2015). Motivación, autoeficacia, estilo atribucional y rendimiento escolar de estudiantes de bachillerato. Revista Electrónica de Investigación Educativa, 17(3), 79-93. 
Bloom, M. (2013). Self-regulated learning: Goal setting and self-monitoring. The Language Teacher, 37(4), 46-51.

Boekaerts, M., \& Corno, L. (2005). Self-Regulation in the Classroom: a perspective on assessment and intervention. Applied Psychology, 54(2), 199-231. Doi: https://doi.org/10.1111/j.1464-0597.2005.00205.x

Broc, M. (2011). Voluntad para estudiar, regulación del esfuerzo, gestión eficaz del tiempo y rendimiento académico en alumnos universitarios. Revista Investigación Universitaria, 29(1), 171-185.

Carranza, R., \& Apaza, E. (2015). Autoconcepto académico y motivación académica en jóvenes talento de una universidad privada de Tarapoto. Propósitos y Representaciones, 3(1), 233-248. Doi: http://dx.doi. org/10.20511/pyr2015.v3n1.72

Castañeda, S., Pineda, M., Gutierrez, E., Romero, N., \& Peñaloza, E. (2010). Construcción de instrumentos de estrategias de estudio, autorregulación y epistemología personal. Validación de constructo. Revista Mexicana de Psicología, 27(1), 77-85.

Chi-Tung, C., \& Ruey-Gwo, C. (2011). The Construction and Verification of a Self-Regulated Learning Process Model of the Electrical Technology Basic Competency. Learning, 5(19), 862-870.

Chilca, M. (2017). Autoestima, hábitos de estudio y rendimiento académico en estudiantes universitarios. Propósitos y Representaciones, 5(1), 7199. Doi: https://doi.org/10.20511/pyr2017.v5n1.145

Cleary, T., Callan, G., \& Zimmerman, B. (2012). Assessing self-regulation as a cyclical, context-specific phenomenon: overview and analysis of SRL microanalytic protocols. Educational Research International, 2012, 1-19. Doi: https://doi.org/10.1155/2012/428639

Durán-Aponte, E., \& Pujol, L. (2013). Manejo del tiempo académico en jóvenes que inician estudios en la Universidad Simón Bolívar. Revista Latinoamericana de Ciencias Sociales, 11(1), 93-108. Doi: https://doi.or $\mathrm{g} / 10.11600 / 1692715 \mathrm{x} .1115080812$

Elvira-Valdéz, M., \& Pujol, L. (2014). Variables cognitivas e ingreso universitario: predictores del rendimiento académico. Universitas Psychologica, 13(4), 1557-1567. Doi: https://doi.org/10.11144/ Javeriana.UPSY13-4.vciu 
Fernández, A., Arnaiz, P., Mejía, R., \& Barca, A. (2015). Atribuciones causales del alumnado universitario de República Dominicana con alto y bajo rendimiento academico. Revista de Estudios e Investigación en Psicología y Educación, 2(1), 19-29. Doi: https://doi.org/10.17979/ reipe.2015.2.1.1319

Fernández, E., Bernardo, A., Suárez, N., Cerezo, R., Núñez, J., \& Rosário, P. (2013). Predicción del uso de estrategias de autorregulación en educación superior. Anales de Psicologia, 29(3), 865-875. https://doi.org/10.6018/ analesps.29.3.139341

Gaeta, M., \& Cavazos, J. (2016). Relación entre tiempo de estudio, autorregulación del aprendizaje y desempeño académico en estudiantes universitarios. Revista de Investigación Educativa, (23), 142-166.

García-Fernández, J., Inglés-Saura, C., Vicent, M., Gonzálvez, C., LagosSan Martín, N., \& Pérez-Sánchez, A. (2016). Relación entre autoeficacia y autoatribuciones académicas en estudiantes chilenos. Universitas Psychologica, 15(1), 79-88. Doi: https://doi.org/10.11144/Javeriana. upsy15-1.raaa

García-Ros, R., \& Pérez-González, F. (2011). Validez predictiva e incremental de las habilidades de autorregulación sobre el éxito académico en la universidad. Revista de Psicodidactica, 16(2), 231-250.

Garello, M., \& Rinaudo, M. (2012). Características de las tareas académicas que favorecen el aprendizaje autorregulado y la cognición distribuida en estudiantes universitarios. Revista de Docencia Universitaria, 10(3), 415-440. Doi: https://doi.org/10.4995/redu.2012.6030

Hadwin, A., \& Oshige, M. (2011). Socially Shared Regulation: Exploring Perspectives of Social in Self-Regulated Learning Theory. Teachers College Record, 113(2), 240-264. Doi: https://doi. org/10.4324/9780203839010.ch5

Hadwin, A., \& Webster, E. (2013). Calibration in goal setting: examining the nature of judgments of confidence. Learning and Instruction, 24(1), 3747. Doi: https://doi.org/10.1016/j.learninstruc.2012.10.001

Heikkila, A., Niemivirta, M., Nieminen, J., \& Lonka, K. (2011). Interrelations among university students' approaches to learning, regulation of learning, and cognitive and attributional strategies: A person oriented approach. 
Higher Education, 61(5), 513-529. Doi: https://doi.org/10.1007/s10734010-9346-2

Inglés, C., Rodríguez-Marín, J., \& González-Pienda, J. (2008). Adaptación de la Sydney Attribution Scale en población universitaria española. Psicothema, 20(1), 166-173.

Macan, T., Shahani, C., Dipboye, R., \& Peek, A. (1990). College Students' Time Management: Correlations With Academic Performance and Stress. Journal of Educational Psychology, 82(4), 760-768. Doi: https:// doi.org/10.1037//0022-0663.82.4.760

Marsh, H. (1984). Relationships among Dimensions of Self-Attribution, Dimensions, of Self-Concept and Academic Achievements. Journal of Educational Psychology, 76(6), 1291-1323. Doi: https://doi.org/https:// doi.org/10.1037/0022-0663.76.6.1291

Muñiz, J., Elosua, P., \& Hambleton, K. (2013). Directrices para la traducción y adaptación de los tests : segunda edición. Psicothema, 25(2), 151-157. Doi: https://doi.org/10.7334/psicothema2013.24

Oettingen, G., Kappes, H., Guttenberg, K., \& Gollwitzer, P. (2015). Selfregulation of time management: mental contrasting with implementation intentions. European Journal of Social Psychology, 45(2), 218-229. Doi: https://doi.org/10.1002/ejsp.2090

Panadero, E. (2017). A review of self-regulated learning: six models and four directions for research. Frontiers in Psychology, 8, 1-28. Doi: https:// doi.org/10.3389/fpsyg.2017.00422

Pérez, E., \& Delgado, M. (2006). Inventario de Autoeficacia Para el Estudio: desarrollo y validación inicial. Avaliação Psicológica, 5(2), 135-143.

Pérez, M., Valenzuela, M., Díaz, A., González-Pienda, J., \& Núñez, J. (2011). Disposición y enfoques de aprendizaje en estudiantes universitarios de primer año. Universitas Psychologica, 10(2), 441-449.

Pérez, M., Valenzuela, M., Díaz, A., González-Pienda, J., \& Núñez, J. (2013). Dificultades de aprendizaje en estudiantes universitarios de primer año. Atenea, 508, 135-150. Doi: http://dx.doi.org/10.4067/ S0718-04622013000200010 
Pintrich, P. (2000). The role of goal orientation in self-regulated learning. En M. Boekaerts, P. Pintrich, \& M. Zeidner (Eds.), Handbook of SelfRegulation (CA: Academ, pp. 452-502). San Diego. Doi: https://doi. org/10.1016/B978-012109890-2/50043-3

Pool-Cibrián, W., \& Martínez-Guerrero, J. (2013). Autoeficacia y uso de estrategias para el aprendizaje autorregulado en estudiantes universitarios. Revista Electrónica de Investigación Educativa, 15(3), 21-37. Recuperado de: http://redie.uabc.mx/vol15no3/contenido-poolmtnez.html

Puustinen, M., \& Pulkkinen, L. (2001). Models of self-regulated learning: a review. Scandinavian Journal of Educational Research, 45(3), 269-286. Doi: https://doi.org/10.1080/00313830120074206

Rosário, P., Mourão, R., Núñez, J., González-Pienda, J., Solano, P., \& Valle, A. (2007). Eficacia de un programa instruccional para la mejora de procesos y estrategias de aprendizaje en la enseñanza superior. Psicothema, 19(3), 422-427.

Schober, B., Klug, J., Jöstl, G., Spiel, C., Dresel, M., Steuer, G., ... Ziegler, A. (2015). Gaining substantial new insights into university students' self-regulated learning competencies: How can we succeed? Zeitschrift für Psychologie., 223(1), 64-65. Doi: https://doi.org/10.1027/21512604/a000201

Sevari, K., \& Kandy, M. (2011). Time management skills impact on selfefficacy and academic performance. Journal of American Science, 7(12), 720-726.

Tauber, S., Dunlosky, J., Rawson, K., Wahlheim, C., \& Jacoby, L. (2012). Self-regulated learning of a natural category: do people interleave or block exemplars during study? Psychonomic Bulletin \& Review, 20(2), 356-363. Doi: https://doi.org/10.3758/s13423-012-0319-6

Torrano, F., Fuentes, J., \& Soria, M. (2017). Aprendizaje autorregulado: estado de la cuestión y retos psicopedagógicos. Perfiles Educativos, 39(156), 160-173.

Tuckman, B. (2003). The effect of learning and motivation strategies training on college students' achievement. Journal of College 
Student Development, 44(3), 430-437. Doi: https://doi.org/10.1353/ csd.2003.0034

Tuckman, B., \& Kennedy, G. (2011). Teaching learning strategies to increase success of first-term college students. The Journal of Experimental Education, 79(4), 478-504. Doi: https://doi.org/10.1080/00220973.201 0.512318

Vives-Varela, T., Durán-Cárdenas, C., Varela-Ruíz, M., \& Fortoul, T. (2014). La autorregulación en el aprendizaje, la luz de un faro en el mar. Investigación en Educación Médica, 3(9), 34-39. Doi: https://doi. org/10.1016/S2007-5057(14)72723-1

Wang, C.-H., Shannon, D., \& Ross, M. (2013). Students' characteristics, self-regulated learning, technology self-efficacy, and course outcomes in online learning. Distance Education, 34(3), 302-323. Doi: https://doi. org/10.1080/01587919.2013.835779

Winne, P., \& Hadwin, A. (1998). Studying as Self-Regulated Learning. En Metacognition in Educational Theory and Practice (pp. 277-304).

Yan, V., Thai, K.-P., \& Bjork, R. (2014). Habits and beliefs that guide selfregulated learning: do they vary with mindset? Journal of Applied Research in Memory and Cognition, 3(3), 140-152. Doi: https://doi. org/10.1016/j.jarmac.2014.04.003

Yusuf, M. (2011). Investigating relationship between self-efficacy , achievement motivation, and self-regulated learning strategies of undergraduate students: a study of integrated motivational models. Procedia Social and Behavioral Sciences, 15, 2614-2617.Doi: https:// doi.org/10.1016/j.sbspro.2011.04.156

Zimmerman, B. (2000). Attaining self-regulation a social cognitive perspective. En M. Boekaerts, P. Pintrich, \& M. Zeidner (Eds.), Handbook of Self-Regulation (San Diego, pp. 13-40). doi: https://doi. org/10.1016/b978-012109890-2/ 50031-7

Zimmerman, B. (2008). Investigating self-regulation and motivation: historical background, methodological developments, and future prospects. American Educational Research Journal, 45(1), 166-183. Doi: https://doi.org/10.3102/0002831207312909 
Zimmerman, B., Bandura, A., Martinez-pons, M., Zimmerman, B., \& Martinez-pons, M. (1992). Self-Motivation for Academic Attainment : The Role of Self-Efficacy Beliefs and Personal Goal Setting. American Educational Research Journal, 29(3), 663-676.

Zimmerman, B., Kitsantas, A., \& Campillo, M. (2005). Evaluación de la autoeficacia regulatoria: una perspectiva social cognitiva. Revista Evaluar, 5, 1-21. Recuperado de http://www.revistaevaluar.com.ar/51. pdf

\section{Agradecimientos:}

Este artículo se realizó en el marco del proyecto de investigación "Impacto de un programa de entrenamiento en estrategias y creencias de disposición al estudio" para optar al grado de doctor en Psicología de la Universidad de Concepción, Chile. Cuenta con financiamiento de Becas Doctorado Nacional CONICYT Folio 21161224; y con apoyo del proyecto FONDECYT "Modelo explicativo de la permanencia y el abandono de los estudios universitarios, basado en procesos cognitivo motivacionales" No 1161502. 